\title{
Case report: optical biopsy in HPV6 lesion
}

The optical biopsy is a noninvasive diagnostic procedure that performs an analysis of the tissue with an optical system that allows reaching a result without extracting the sample (excisional biopsy) from the body tissue. Particularly, we are making spectrofluorometry (SFM) observations in loci lesions and healthy surrounding areas. The tissue is accessed through the body surface, including the analysis of the skin itself or mucosal surface, or by endoscope into any cavity. We have found spectral similarities in the detection of infectious diseases of their natural spectral fluorescence. Specifically, in the analyzed cases of DNA-HPV ${ }^{+}$patients, we observed in all of their fluorescence spectra the same type of relevant peaks. So, we present here the SFM observation of HPV6 infected hard palate case. In addition, we show in this case the positive reaction images after the topication with 3-5\% acetic acid as it occurs in the cervix uterine lesion.

\section{KEYWORDS: fluorescence - autofluorescence - spectral analysis - HPV - diagnosis}

\section{Introduction}

Our prolonged experience collecting valuable data through the professional and academic employment of ionizing sources of radiations and emissions of lasers and LEDs in surgical, treatment and diagnostic procedures, allows us to identify some interesting niches of physics medical research which, in turn, brings us enough appropriate results to develop a clinical tool to search on the healthy state of the population [1-9]. Then, we will focus on noninvasive optics analytical techniques we extensive used in laboratory applications as well as in medical practice. In this case we are referring to the spectral analysis of the fluorescence emitted from tissues due to the laser or LED light excitation or spectrofluorometry (SFM). SFM will allow for in-vivo real time diagnoses (optical biopsy) without removal of a tissue sample or treatment with a contrast photosensitizer drugs (photodynamic diagnosis) in almost every type of cancer and early neoplastic difference observed in humans, particularly in cases of HPV infections, intraepithelial progressions to cancer and different stages of cancer in urogenital tract, anus, oral and skin sites. We observed the SFM using an appropriate dual-pass fiber optics 0.20 $\mathrm{mm}$ in diameter, which allows simultaneous local excitations of selected points at $400 \mathrm{~nm}$ and fluorescence emission measurements in the 500-800 $\mathrm{nm}$ range.

\section{Case Report}

In the last four years we have found spectral similarities in the detection of cancer and infectious diseases of their natural fluorescence. Specifically, in the few analyzed cases of DNA$\mathrm{HPV}^{+}$patients, we observed in all of their fluorescence spectra the same type of relevant peaks. The in-situ fluorescence analysis is related with biochemical and molecular changes in the affected tissues of patients. Due to that, by comparing the fluorescence spectra of healthy tissue and infected tissue we can infer that some HPV genotype is present. In this case, the autofluorescence spectra were taken on hard palate of a lesion caused by HPV6, which was previously diagnosed by molecular tests (Hybrid Capture CH2) and the "gold standard" (conventional biopsy). The Figure 1 shown the sampled points in the hard palate of a patient affected by HPV6 (a) and the spectra obtained by SFM ( $b$ and $c$ ). The three black tested points were selected inside the small almost rectangular section $\left(0.5 \times 1.5 \mathrm{~cm}^{2}\right)$ easily recognized at naked eye from its surrounding area due to the intensity of its reddish color; obviously this section was identified as clinically suspicious. Correspondingly, their fluorescence signals are represented in black traces. The other four tested points (two colored in red and the others in blue) were selected in the considered healthy area, located at distances of the order of a half to one centimeter to the suspicious section. As before, their fluorescence signals are represented in c) in red and blue traces, but surprisingly in the case of the blue traces they look like the black traces in b). Then, it means that around the blue points the HPV6 infection is just interstitially present but its macroscopic effect is not yet observed

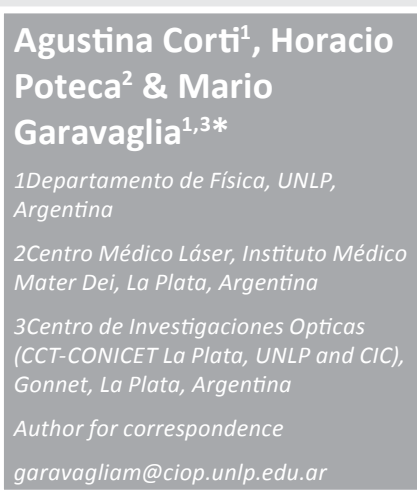



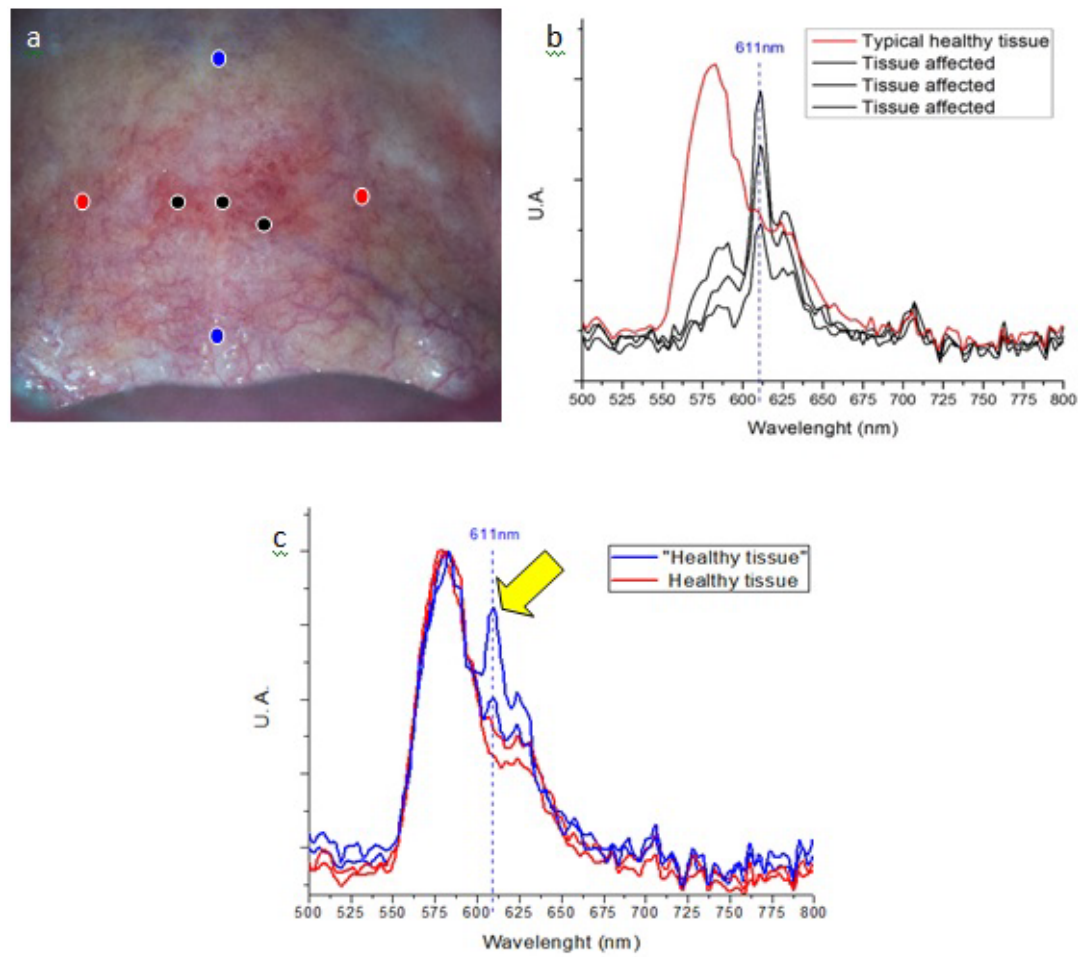

Figure 1. Sampled points in the hard palate of a patient affected by HPV6 (a) and the spectra obtained by SFM (b and c).
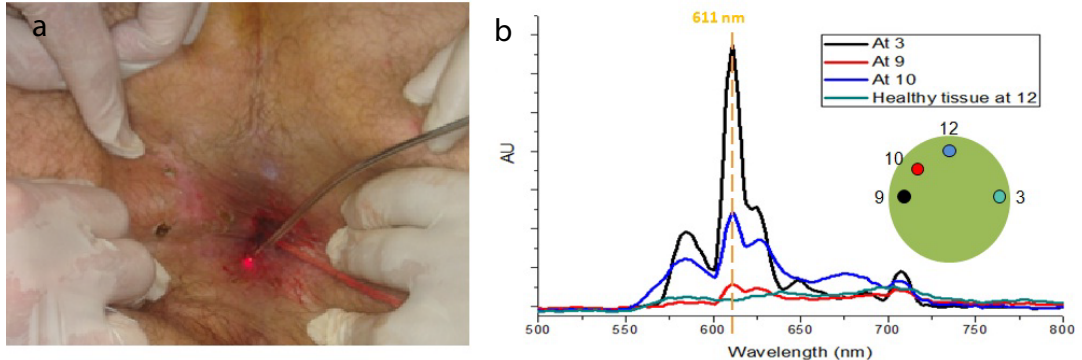

Figure 2. Observation of the warts autofluorescence spectra figure $2 \mathrm{~b}$ and then their laser photocoagulation and photo-denaturation follow.

by sight. This observational fact is a positive argument to consider fluorescence biopsies as a prior complementary clinical technique to the conventional biopsy.

As it was shown in Figure 1b, the fluorescence spectra of palatal regions affected by HPV6 show a characteristic peak at $611 \mathrm{~nm}$, while the spectra of their healthy areas are typical of it. Apparently that peak seems to be part of the signature of the HPV6 infection. To support this assumption, we include the case of another patient who has warts due to HPV6 in the perianal area. Figure 2a show photography of the patient during a surgical procedure which starts with the observation of the warts autofluorescence spectra Figure $2 b$ and then their laser photocoagulation and photo-denaturation follow. The autofluorescent samples depicted as in the clock positions 3,9 and 10 correspond to pathological perianal HPV6 lesions, while 12 is the control sample taken on healthy skin which differs from the SFM of the hard palate mucous. It is clear that although pathological signals are affected in its intensity due to body topography (angular factor effect observation) all of them have similar structure and show the characteristic peak at $611 \mathrm{~nm}$.

Besides, in this studied case it was observed a positive reaction topication with $3-5 \%$ acetic acid, as it is shown in the right photo of Figure 3 as it occurs in the cervix uterine lesion [9]. This is another interesting observational fact of a classical gynecological optical biopsy (acetic white) that could be translated to oral optical biopsy. 

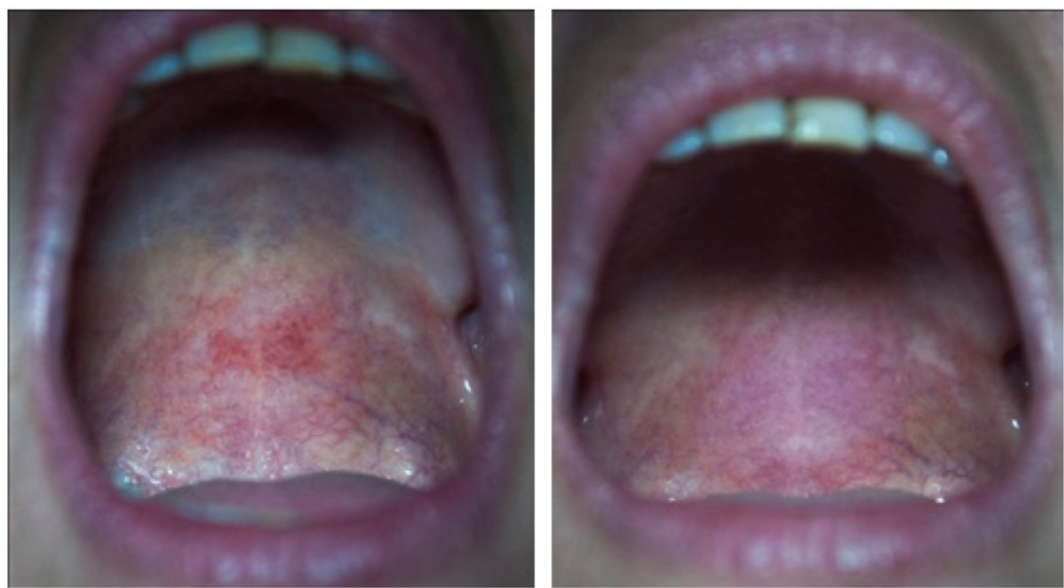

Figure 3. Oral optical biopsy

\section{Discussion}

The SFM of a cancer tissue and surrounding normal tissue differ in their characteristic spectra [10]. The present work shows that this is also true for the case of HPV infection. SFM makes comparative observations of the natural fluorescence spectra of a suspicious area and its neighbor healthy area, allowing us to propose to make a triage. So, this study would be innovative in the field of gynecology, infectology, and oncology since it is a non-invasive diagnostic procedure which allows exploring tissues (skin and mucous membranes) several millimeters in depth. In addition, note the peak that appears at $611 \mathrm{~nm}$ accompanying the spectrum of the shots taken in areas of "healthy tissue" according to a naked eye observation by a trained dentist, indicating the increased sensitivity of the technique, detecting infected areas whose appearance looks like normal.

Then, in principle, the SFM as well as the colposcopy produced the same results in terms of "lesion location" (acetowhite), but what is more important in SFM is that it will provide information about the active neoplastic process in-situ.

\section{REFERENCES}

1. Agustina C, Mario $G_{\text {th }}$ Optical biopsy. Some preliminary results. $16^{\text {th }}$ International Congress on Photobiology. Universidad Nacional de Córdoba, Córdoba, Argentina. 7-12, 2014.

2. Etcheverry ME, Pasquale, Miguel A et al. Comparison in vitro of the photodynamic action of temoporfin using 637 and $400 \mathrm{~nm}$ excitation sources. $16^{\text {th }}$ International Congress on Photobiology. Universidad Nacional de Córdoba, Córdoba, Argentina. September 7-12, 2014.

3. Estela IA, Etcheverry ME, Agustina C et al. Photobiology in hpv intraepithelial lesions: photodynamic diagnostic and photodynamic therapy. ICGEB Workshop Human Papillomavirus: From Basic Biology to Cervical

\section{Cancer Prevention”. 19-21, 2014.}

4. Estela IA, Eycheverry ME, Agustina C et al. Scop and photodynamic therapy in tissues infected by hpv. ICGEBIDNATumour Virus Meeting 2015. 21-25, 2015.

5. Agustina C, Miguel AP, Estela IA. Applicability of fluorescence spectroscopy in urine samples for detecting oncogenic activity, 2016.

6. Agustina C, Etcheverry ME, EstelaI IA et al. Optical biopsies: thermal infrared and visible fluorescent images from normal and cancerous tissues. Photodynamic diagnostic and photodynamic therapy, 2016.

7. Etcheverry ME, Pasquale MA, Garavaglia M. Photodynamic therapy of hela cell cultures by using led or laser sources. J. Photochem. Photobiol. B. 160, 271-277, 2016.
8. Etcheverry ME, Agustina C, Solange B et al. Pasquale and Mario Garavaglia. Infrared imaging and fluorescence measurements in photodynamic therapy of a fibrosarcome animal mode. Revista. Medicina. 77, 519-520, 2017.

9. Eugenia EM, Ángel PM, Anabella G et al. Photodynamic therapy in fibrosarcome Balb/C animal mode: observation of Rebound effect. Photodiagnosis. Photodyn. Ther. 21, 98-107, 2018.

10. Haripriya V, Michelle I. Silver, B. Kalpana et al. Determinants of VIA (Visual Inspection of the Cervix After Acetic Acid Application) Positivity in Cervical Cancer Screening of Women in a Peri-Urban Area in Andhra Pradesh, India. Cancer. Epidemiol. Biomarkers. Prev. 19, 13731380 (2010). 